Обоснование. Полиморфизм rs 1801282 (Pro12Ala) может являться одной из причин неоднородного ответа пациентов с нарушениями углеводного обмена на терапию тиазолидиндионами. Исследование данного полиморфизма у пациентов с метаболическим синдромом (MC) поможет выделить группу пациентов, у которых целесообразно применение агонистов PPAR- $\gamma$.

Цель. Оценить клинический эффект применения тиазолидиндионов у пациентов с нарушениями углеводного обмена в зависимости от наличия полиморфизма гена PPAR-ү rs 1801282.

Методы. В открытое когортное исследование были включены все пациенты с впервые выявленным МС с нарушениями углеводного обмена, не получавшие ранее сахароснижающей терапии. Всем пациентам рекомендовали диету, расширение физической активности и пиоглитазон в дозе 30 мг в день - селективный агонист PPAR- $ү$-рецептора. После назначения терапии пациенты являлись в центр повторно через 12 недель.

В качестве основного исхода в исследовании оценивали у пациентов с нарушением толерантности к глюкозе (НТГ) гликемию натощак и через 2 ч после нагрузки глюкозой, у пациентов с сахарным диабетом 2 типа (СД2) - уровень гликированного гемоглобина $\left(\mathrm{HbA}_{1 \mathrm{c}}\right)$.

Результаты. В исследование были включены 109 пациентов. Из них у 14 пациентов было выявлено носительство полиморфизма rs1801282, у остальных 95 был определен типичный генотип PPAR чения терапии в группах НТГ и СД2 наблюдалось улучшение гликемического контроля. Степень снижения уровня глюкозы плазмы натощак и после нагрузки глюкозой была более выражена при НТГ у пациентов с полиморфизмом rs1801282 в сравнении с остальными (уровень глюкозы плазмы натощак составил -0,7 [-0,9; -0,7] против -0,4 [-0,5; -0,3] ммоль/л, p=0,001; уровень глюкозы плазмы через 2 ч после нагрузки глюкозой составил $-1,1[-1,8 ;-0,3]$ против -0,5 [-0,7; -0,1] ммоль/л, p=0,031). У пациентов с СД2 не получено данных за статистически значимое влияние полиморфизма rs 1801282 на результаты применения пиоглитазона, однако отмечалась тенденция к большему снижению глюкозы плазмы натощак в случае носительства полиморфного гена $(-1,9[-2,2 ;-1,8]$ против -1,5 [-1,7; -1,2] ммоль/л, $\mathrm{p}=0,073)$.

Заключение. Проведенное исследование показывает влияние полиморфизма rs 1801282 на результаты применения пиоглитазона как у пациентов с НТГ, так и при СД2. Носительство полиморфизма приводит к существенному уменьшению гликемии натощак и после нагрузки глюкозой у пациентов с НТГ. Тенденция к улучшению показателей углеводного обмена (гликемия натощак, $\mathrm{HbA}_{1 c}$ ) отмечена в подгруппе больных СД2.

КЛЮЧЕВЫЕ СЛОВА: метаболический синдром; сахарный диабет 2 типа; нарушение толерантности к глюкозе; тиазолидиндионы; полиморфизм rs1801282 (Pro12Ala).

\title{
CLINICAL EFFECT OF THIAZOLIDINEDIONES IN SUBJECTS WITH DISORDERS OF CARBOHYDRATE METABOLISM IN CASE OF POLYMORPHISM RS1801282
}

\author{
(c) Tatyana V. Eremenko*, Nikolay A. Matsievskiy, Natalya V. Vorokhobina, Irina Y. Matezius, Irina N. Abramashvili
}

North-western State Medical University named after I.I. Mechnikov

BACKGROUND: The polymorphism rs1801282 (Pro12Ala) may be one of the reasons for the heterogeneous response of patients with carbohydrate metabolism disorders to thiazolidinedione therapy. Studies of this polymorphism in patients with metabolic syndrome (MS) will help identify a group of patients in whom the use of thiazolidinedione is advisable.

AIMS: To assess the clinical effect of thiazolidinediones in patients with metabolic syndrome, depending on the presence of polymorphism rs1801282.

MATERIALS AND METHODS: All patients with newly diagnosed MS with impaired carbohydrate metabolism were included in the open cohort study. All patients were recommended a diet, expansion of physical activity and pioglitazone at a dose of $30 \mathrm{mg}$ per day. After the appointment of the therapy, the patients come to the center back at 12 weeks.

The main outcome in the study assessed in patients with impaired glucose tolerance (IGT) was fasting glycemia and 2 hours after glucose tolerance test, in patients with type 2 diabetes $-\mathrm{HbA}_{1 c}$. 
RESULTS: 109 patients were included in the study. Of these, 14 were carriers of rs1801282, the other 95 had a typical PPARY genotype. After the appointment of therapy in the groups of IGT and type 2 diabetes, improvement of glycemic control was observed. The degree of decrease in fasting plasma glucose and after glucose tolerance test was more pronounced with IGT in patients with polymorphism rs1801282 compared with the rest (plasma fasting plasma glucose level was $-0.7[-0.9,-0.7]$ vs. $-0,4[-0.5,-0.3] \mathrm{mmol} / \mathrm{L}, \mathrm{p}=0.001 ;$ plasma glucose level 2 hours after glucose tolerance test was $-1.1[-1.8,-0.3]$ vs. $-0.5[-0.7,-0.1] \mathrm{mmol} / \mathrm{L}, \mathrm{p}=0.031)$. In patients with type 2 diabetes, no data were obtained for the statistically significant effect of rs1801282 polymorphism on the results of pioglitazone, but there was a tendency for a greater decrease in fasting plasma glucose in the case of carrying the polymorphic gene $(-1.9[-2.2,-1.8]$ against $-1,5[-1,7,-1,2] \mathrm{mmol} / \mathrm{l}, \mathrm{p}=0,073)$.

CONCLUSIONS: The study shows the effect of polymorphism rs1801282 on the results of pioglitazone in patients with MS, both in IGT and in type 2 diabetes. Carrying polymorphism leads to a significant decrease in fasting glycemia and after glucose tolerance test in patients with IGT. The tendency to improve the parameters of carbohydrate metabolism (fasting glycemia, $\mathrm{HbA}_{1 c}$ ) was noted in a subgroup of patients with type 2 diabetes.

KEYWORDS: metabolic syndrome; diabetes mellitus type 2; glucose intolerance; thiazolidinediones; polymorphism rs 1801282 (Pro12Ala).

\section{ОБОСНОВАНИЕ}

Хорошо известно о том, что рецептор, активируемый пролифераторами пероксисом гамма (Peroxisome proliferator-activated receptor gamma [PPARү]), регулирует экспрессию генов, играющих ключевую роль в процессах накопления и мобилизации липидов, метаболизма глюкозы, воспаления, а нарушение регуляции PPAR связано с развитием ожирения, сахарного диабета 2 типа (СД2), атеросклероза и метаболического синдрома (МС) [1]. Возможности терапии агонистами PPARү могут быть эффективным патогенетическим подходом в терапии ряда состояний, в том числе СД2 и МС. Тем не менее результаты исследований показывают, что не у всех пациентов эта группа препаратов оказывается эффективной. Так, например, среди пациентов с СД2 у трети пациентов пиоглитазон оказывается неэффективным [2].

В связи с этим изучение возможностей тиазолидиндионов в отдельных когортах пациентов и выделение целевой группы больных представляется важной задачей современной медицины.

Несомненный интерес также представляет эффект пиоглитазона у пациентов с НТГ. Исследование АCT NOW показало, что назначение пиоглитазона при НТГ сопровождается уменьшением риска развития СД2 с 7,6\% до 2,1\%, а также уменьшением диастолического АД, риска утолщения интимы a. carotis, увеличением уровня липопротеидов высокой плотности (ЛПВП) [3].

Существует ряд полиморфизмов гена $P P A R \gamma$, которые приводят к выраженному снижению активности этого белка. Наиболее частым из них является полиморфизм гена PPARy2 rs1801282 (Pro12Ala). Его распространенность в популяции, согласно результатам крупного метаанализа, составляет 9\% [4]. Учитывая столь высокую встречаемость, полиморфизм rs1801282 может являться одной из причин неоднородного ответа пациентов с нарушениями углеводного обмена на терапию тиазолидиндионами, поэтому изучение эффекта препаратов этой группы у пациентов-носителей rs1801282 представляет научный и практический интерес.

\section{ЦЕЛЬ}

Оценить клинический эффект применения тиазолидиндионов у пациентов с нарушениями углеводного обмена в зависимости от наличия полиморфизма rs 1801282.

\section{МЕТОДЫ}

\section{Дизайн исследования}

Проведено открытое одноцентровое проспективное интервенционное выборочное когортное клиническое исследование.

Критерии соответствия

Критерии включения: впервые выявленный МС с нарушениями углеводного обмена, пациенты ранее не принимали гиполипидемические препараты. Другие специальные критерии включения/невключения не применялись.

Критерии исключения: непереносимость пиоглитазона, низкая приверженность лечению, наличие противопоказаний к препарату, а также обстоятельства, не позволявшие пациенту являться в центр для обследования.

\section{Условия проведения}

Набор пациентов проводили в клинике кафедры эндокринологии им. акад. В.Г. Баранова ФГБОУ ВО «Северо-Западный государственный медицинский университет имени И.И. Мечникова» Министерства здравоохранения РФ.

\section{Продолжительность исследования}

Запланированная продолжительность периода включения в исследование составила 2 года. После назначения терапии пациенты являлись в центр повторно через 6 и 12 недель или ранее при возникновении клинической необходимости. Пациентам, прекратившим терапию по медицинским показаниям, проводился дополнительный визит через 4 недели после прекращения лечения. Исследование проводилось с 01.09.2014 по 01.09.2016 гг.

\section{Описание медицинского вмешательства}

Всем пациентам до назначения терапии проводили обследование, включавшее сбор анамнеза, определение антропометрических (в т. ч. измерение роста, веса) и лабораторных показателей. Оценивали уровень гликемии натощак и постпрандиальный, проводили пероральный глюкозотолерантный тест в сомнительных случаях и определение уровня гликозилированного гемоглобина $\left(\mathrm{HbA}_{1 c}\right)$. В рамках проведенного исследования у всех пациентов проводилось генотипирование полиморфизма rs1801282. 
После обследования для коррекции нарушений углеводного обмена всем пациентам рекомендовали диету, расширение физической активности и пиоглитазон в дозе 30 мг в день. Через 6 недель пациент в плановом порядке являлся в центр для сбора данных по жалобам, уровню гликемии и коррекции диеты. На визите через 12 недель у пациента повторно оценивали жалобы, антропометрические и лабораторные (гликемия, $\mathrm{HbA}_{1 c}$ ) показатели. Пациентам с нарушенной толерантностью к глюкозе (НТГ) проводили повторно пероральный глюкозотолерантный тест. На дополнительном визите через 4 недели после отмены препарата у пациентов оценивали наличие жалоб.

\section{Основной исход исследования}

В качестве основного исхода в исследовании оценивали состояние углеводного обмена. Для этой цели у пациентов с НТГ оценивали гликемию натощак и через 2 ч после нагрузки глюкозой, у пациентов с СД2 - уровень $\mathrm{HbA}_{1 \mathrm{c}}$.

\section{Анализ в подгруппах}

Группы в исследовании формировались в зависимости от наличия полиморфизма rs1801282. В каждой из групп анализировались подгруппы пациентов, имеющих пограничные нарушения углеводного обмена (НТГ) или явный СД2.

\section{Методы регистрации исходов}

MC диагностировали согласно совместным критериям Международной федерации диабета, Национального института сердца, легких и крови, Американской кардиологической ассоциации, Всемирной кардиологической федерации, Международного общества атеросклероза и Международной ассоциации по изучению ожирения [5]. Согласно этим критериям, диагноз устанавливали при одновременном наличии трех и более критериев из нижеследующих:

- окружность талии $\geq 94$ см у мужчин, $\geq 80$ см у женщин (значения взяты для европеоидов);

- триглицериды $\geq 1,7$ ммоль/л или гиполипидемическая терапия;

- ЛПВП <1,0 ммоль/л у мужчин, <1,3 ммоль/л у женщин;

- глюкоза плазмы натощак 25,6 ммоль/л или сахароснижающая терапия;

- артериальная гипертензия: систолическое артериальное давление $\geq 130$ и/или диастолическое артериальное давление $\geq 85$ мм рт. ст. или антигипертензивная терапия.

Для оценки гликемии использовали персональные глюкометры Accu-Chek Performa и соответствующие тест-полоски. Для оценки $\mathrm{HbA}_{1 с}$ использовали портативный анализатор A1cNow и соответствующие расходные материалы. Для измерения антропометрических показателей у пациентов использовали ростомер и весы «Масса-К ВЭМ-150-»Масса-К» АЗ».

Амплификация фрагментов ДНК проводилась методом полимеразно-цепной реакции с использованием специфических праймеров, анализ продуктов проводили после электрофореза в полиакриламидном геле.
Этическая экспертиза.

Выписка из протокола № 9 заседания Локального этического комитета ФГБО ВО СЗГМУ им. И.И. Мечникова, заседание 03.10.2018. Научное исследование соответствует международным этическим нормам, изложенным В Хельсинской декларации Всемирной медицинской ассоциации «Рекомендации для врачей, занимающихся биомедицинскими исследованиями»

\section{Статистический анализ}

Принципы расчета размера выборки. Размер выборки предварительно не рассчитывался.

Методы статистического анализа данных. Для статистического анализа использовали программный пакет Statistica 8.0 (Statsoft, США). Описательная статистика представлена с указанием медианы и первой и третьей квартилей: Ме [Q1; Q3]. Статистическая обработка проводилась с использованием непараметрических методов: для сравнения независимых показателей использовался критерий Манна-Уитни, для сравнения зависимых критерий Вилкоксона, категориальные данные анализировались с использованием точного двустороннего критерия Фишера ввиду небольшого количества наблюдений.

\section{РЕЗУЛЬТАТЫ}

Объекты (участники) исследования

В исследование было включено 109 пациентов с впервые выявленным MC с гипергликемией натощак. Из них у 14 пациентов было выявлено носительство полиморфизма rs1801282, у остальных 95 был определен типичный генотип PPAR- $\gamma$. В таблице 1 представлено распределение пациентов в каждой группе по состоянию углеводного обмена по результатам проведенного обследования. В таблице 2 представлены основные демографические характеристики пациентов и показатели углеводного обмена в зависимости от формы нарушения углеводного обмена. Антигипертензивную терапию получали 49 пациентов, прием был системным, у 9 пациентов потребовалась коррекция гипотензивной терапии в ходе исследования. Гиполипидемическую терапию пациенты не получали, однако некоторым потребовалось назначение статинов или фибратов после окончания исследования.

Таблица 1. Состояние углеводного обмена у больных в зависимости от наличия полиморфизма rs 1801282

\begin{tabular}{lcc}
\hline $\begin{array}{c}\text { Пациенты с } \\
\text { полиморфизмом } \\
\text { rs 1801282 } \\
(\mathbf{n = 1 4 )}\end{array}$ & $\begin{array}{c}\text { Пациенты без } \\
\text { полиморфизма } \\
\text { rs1801282 } \\
\text { (n=95) }\end{array}$ \\
\hline НТГ & $9(64,3 \%)$ & $58(61,1 \%)$ \\
СД2 & $5(35,7 \%)$ & \\
\hline
\end{tabular}


Таблица 2. Демографическая характеристика и исходные показатели углеводного обмена у больных в зависимости от формы нарушения углеводного обмена и наличия полиморфизма rs 1801282

\begin{tabular}{|c|c|c|c|c|c|c|}
\hline & \multicolumn{3}{|c|}{ Пациенты с НТГ } & \multicolumn{3}{|c|}{ Пациенты с СД2 } \\
\hline & $\begin{array}{c}\text { Носительство } \\
\text { rs1801282 } \\
(n=9)\end{array}$ & $\begin{array}{c}\text { Типичный } \\
\text { генотип PPARY } \\
(n=58)\end{array}$ & $\mathbf{p}$ & $\begin{array}{c}\text { Носительство } \\
\text { rs } 1801282 \\
(n=5)\end{array}$ & $\begin{array}{c}\text { Типичный } \\
\text { генотип PPARY } \\
(\mathrm{n}=37)\end{array}$ & $\mathbf{p}$ \\
\hline Пол, м/ж & $5 / 4$ & $41 / 17$ & $0,446^{*}$ & $2 / 3$ & $23 / 14$ & $0,380^{*}$ \\
\hline Возраст, годы & $58[51 ; 60]$ & $52[50 ; 57]$ & $0,279^{* *}$ & $59[55 ; 63]$ & $53[51 ; 57]$ & $0,037^{* *}$ \\
\hline Вес, кг & $111,1[98,0 ; 115,9]$ & $104,2[97,4 ; 110,2]$ & $0,248^{* *}$ & $89,2[86,3 ; 106,5]$ & $103,2[94,3 ; 112,1]$ & $0,193^{* *}$ \\
\hline ИМТ, кг/м² & $36[34 ; 38]$ & $34[32 ; 36]$ & $0,067^{* *}$ & $35[35 ; 36]$ & $34[33 ; 35]$ & $0,155^{* *}$ \\
\hline $\begin{array}{l}\text { Глюкоза плазмы } \\
\text { натощак, ммоль/л }\end{array}$ & $5,9[5,7 ; 5,9]$ & $5,9[5,7 ; 6,0]$ & $0,304^{* *}$ & $6,9[6,9 ; 7,1]$ & $9,3[8,2 ; 9,9]$ & $<0,001^{* *}$ \\
\hline $\begin{array}{l}\text { Глюкоза плазмы чер } \\
2 \text { ч после нагрузки } \\
\text { глюкозой, ммоль/л }\end{array}$ & $9,6[8,2 ; 9,8]$ & $9,5[8,9 ; 10,4]$ & $0,556^{* *}$ & - & - & - \\
\hline $\mathrm{HbA}_{1 c^{\prime}} \%$ & 一 & 一 & 一 & $7,3[7,2 ; 7,4]$ & $8,3[7,4 ; 8,9]$ & $0,023^{* *}$ \\
\hline
\end{tabular}

Примечание. * - критерий статистической значимости при использовании точного критерия Фишера; ** - то же при использовании критерия Манна-Уитни

Основные результаты исследования

Результаты терапии пиоглитазоном у пациентов оценивались через 3 месяца в подгруппах соответственно форме нарушений углеводного обмена и наличию полиморфизма rs1801282. Основные результаты приведены в таблицах 3 и 4.

Приведенные данные свидетельствуют о том, что назначение терапии пиоглитазоном в дозе 30 мг суще- ственно улучшало состояние углеводного обмена у пациентов в группах НТГ и СД2 как с полиморфизмом, так и без него: наблюдалось статистически значимое снижение гликемии натощак и после нагрузки глюкозой, а также уровня $\mathrm{HbA}_{1 c^{\circ}}$

Для сравнения результатов терапии в группах пациентов с полиморфизмом rs1801282 и без него были

Таблица 3. Показатели углеводного обмена у пациентов при носительстве полиморфизма rs 1801282 до лечения и через 3 месяца после

\begin{tabular}{|c|c|c|c|c|c|c|}
\hline & \multicolumn{3}{|c|}{$\begin{array}{c}\text { Пациенты-носители полиморфизма } \\
\text { rs } 1801282 \text { с НTГ }(\mathrm{n}=9)\end{array}$} & \multicolumn{3}{|c|}{$\begin{array}{c}\text { Пациенты-носители полиморфизма } \\
\text { rs } 1801282 \text { с СД2 (n=5) }\end{array}$} \\
\hline & До лечения & Через 3 мес & p* & До лечения & Через 3 мес & $\mathbf{p}^{*}$ \\
\hline $\begin{array}{l}\text { Глюкоза плазмы натощак, } \\
\text { ммоль/л }\end{array}$ & $5,9[5,7 ; 5,9]$ & $5,0[4,9 ; 5,2]$ & 0,011 & $6,9[6,9 ; 7,1]$ & $5,2[5,0 ; 5,2]$ & 0,043 \\
\hline $\begin{array}{l}\text { Глюкоза плазмы через } 2 \text { ч после } \\
\text { нагрузки глюкозой, ммоль/л }\end{array}$ & $9,6[8,2 ; 9,8]$ & $7,7[7,4 ; 8,3]$ & 0,008 & - & - & - \\
\hline $\mathrm{HbA}_{1 c^{\prime}} \%$ & - & - & - & $7,3[7,2 ; 7,4]$ & $6,6[6,3 ; 6,6]$ & 0,043 \\
\hline
\end{tabular}

Примечание. * - критерий статистической значимости при использовании критерия Вилкоксона для связанных показателей.

Таблица 4. Показатели углеводного обмена у пациентов без носительства полиморфизма rs1801282 до лечения и через 3 месяца после

\begin{tabular}{|c|c|c|c|c|c|c|}
\hline & \multicolumn{3}{|c|}{$\begin{array}{c}\text { Пациенты без полиморфизма } \\
\text { rs1801282 с НТГ (n=58) }\end{array}$} & \multicolumn{3}{|c|}{$\begin{array}{c}\text { Пациенты без полиморфизма } \\
\text { rs1801282 с СД2 (n=37) }\end{array}$} \\
\hline & До лечения & Через 3 мес & $\mathbf{p}^{*}$ & До лечения & Через 3 мес. & $\mathbf{p}^{*}$ \\
\hline $\begin{array}{l}\text { Глюкоза плазмы натощак, } \\
\text { ммоль/л }\end{array}$ & $5,9[5,7 ; 6,0]$ & $5,5[5,3 ; 5,6]$ & $<0,001$ & $9,3[8,2 ; 9,9]$ & $8,0[7,1 ; 8,3]$ & $<0,001$ \\
\hline $\begin{array}{l}\text { Глюкоза плазмы через } 2 \text { часа после } \\
\text { нагрузки глюкозой, ммоль/л }\end{array}$ & $9,5[8,9 ; 10,4]$ & $9,2[8,5 ; 9,8]$ & $<0,001$ & - & - & - \\
\hline $\mathrm{HbA}_{1 c^{\prime}} \%$ & - & - & - & $8,3[7,4 ; 8,9]$ & $7,4[6,7 ; 8,0]$ & $<0,001$ \\
\hline
\end{tabular}

Примечание. * - критерий статистической значимости при использовании критерия Вилкоксона для связанных показателей. 
Таблица 5. Изменения показателей углеводного обмена у пациентов в зависимости от носительства полиморфизма rs 1801282 через 3 месяца терапии

\begin{tabular}{|c|c|c|c|c|c|c|}
\hline & \multicolumn{3}{|c|}{ Пациенты с НТГ } & \multicolumn{3}{|c|}{ Пациенты с СД2 } \\
\hline & $\begin{array}{l}\text { Носительство } \\
\text { rs1801282 } \\
(\mathrm{n}=9)\end{array}$ & $\begin{array}{l}\text { Типичный } \\
\text { генотип PPARY } \\
(\mathrm{n}=58)\end{array}$ & $\mathbf{p}^{*}$ & $\begin{array}{l}\text { Носительство } \\
\text { rs1801282 } \\
(\mathrm{n}=5)\end{array}$ & $\begin{array}{l}\text { Типичный } \\
\text { генотип PPARY } \\
(\mathrm{n}=37)\end{array}$ & $\mathbf{p}^{*}$ \\
\hline $\begin{array}{l}\text { Изменение глюкозы плазмы } \\
\text { натощак, мМоль/л }\end{array}$ & $-0,7[-0,9 ;-0,7]$ & $-0,4[-0,5 ;-0,3]$ & 0,001 & $-1,9[-2,2 ;-1,8]$ & $-1,5[-1,7 ;-1,2]$ & 0,073 \\
\hline $\begin{array}{l}\text { Изменение глюкозы плазмы } \\
\text { через } 2 \text { часа после нагрузки } \\
\text { глюкозой, мМоль/л }\end{array}$ & $-1,1[-1,8 ;-0,3]$ & $-0,5[-0,7 ;-0,1]$ & 0,031 & - & - & - \\
\hline Изменение $\mathrm{HbA}_{1 c^{\prime}} \%$ & - & - & - & $-0,8[-0,9 ;-0,7]$ & $-0,7[-0,8 ;-0,6]$ & 0,133 \\
\hline
\end{tabular}

Примечание. * - критерий статистической значимости при использовании точного критерия Манна-Уитни.

сопоставлены изменения контролируемых показателей состояния углеводного обмена. Результаты приведены в таблице 5.

Как видно из представленных данных, степень снижения глюкозы плазмы натощак и после нагрузки глюкозой была более выражена при НТГ у пациентов с полиморфизмом rs 1801282 в сравнении с остальными.

У пациентов с СД2 не получено данных за статистически значимое влияние полиморфизма rs1801282 на результаты применения пиоглитазона, однако отмечалась тенденция к большему снижению глюкозы плазмы натощак в случае носительства полиморфного гена.

\section{Нежелательные явления}

При применении пиоглитазона нередко встречаются отечность и набор веса. Из 109 пациентов отечность отмечена у 8 (7,3\%) пациентов, набор веса более 3 кг у 12 (11,0\%). Данные нежелательные явления послужили причиной отмены пиоглитазона после окончания исследования у 3 пациентов по причине отечности и у 9 по причине набора веса.

Также за время исследования отмечены: головная боль у 4 пациентов, симптоматическая гипогликемия у одного пациента и миалгия у одного пациента. Данные нежелательные явления носили транзиторный характер и купировались самостоятельно, не потребовав отмены проводимой терапии или коррекции дозы на протяжении исследования.

Отмечено одно серьезное нежелательное явление впервые возникший пароксизм фибрилляции предсердий. Пациенту назначена терапия по поводу заболевания сердца, пиоглитазон отменен не был.

Все указанные нежелательные явления являются ожидаемыми и не меняют представления о безопасности применения пиоглитазона у пациентов с нарушениями углеводного обмена. Не обнаружено данных, указывающих на влияние полиморфизма rs1801282 на риск нежелательных явлений при использовании пиоглитазона.

\section{ОБСУЖДЕНИЕ}

Резюме основного результата исследования

Существующие в настоящее время научные сведения по влиянию полиморфизма rs 1801282 на эффективность применения глитазонов весьма противоречивы. В нашей работе на фоне терапии пиоглитазоном состояние углеводного обмена улучшилось у всех подгрупп пациентов. У пациентов с полиморфизмом rs 1801282 при НТГ влияние терапии на показатели углеводного обмена было более выражено, чем у пациентов с типичным типом PPAR- $\gamma$. Различия в подгруппах пациентов с HTГ при наличии полиморфизма и без него достигли статистической значимости. Аналогичная динамика отмечалась и среди пациентов с СД2, однако статистической значимости различия в подгруппах не достигли. Таким образом, результаты проведенного исследования убедительно демонстрируют, что активность пиоглитазона зависит от наличия полиморфизма rs 1801282. Результаты исследования свидетельствуют о том, что у пациентов с полиморфизмом rs1801282 и МС с нарушениями углеводного обмена использование тиазолидиндионов может быть патогенетически обосновано. Терапия пиоглитазоном в целом характеризовалась благоприятным профилем безопасности

Обсуждение основного результата исследования

В настоящее время существует ограниченное количество данных о влиянии полиморфизма rs1801282 на результаты применения тиазолидиндионов. Возможности пиоглитазона у пациентов с нарушенной толерантностью к глюкозе наиболее наглядно демонстрируются результатами исследования АCT NOW. Согласно результатам исследования, применение препарата снизило риск развития СД2 на 72\%. Конверсия в нормальный гликемический статус произошла у 48\% пациентов, принимающих пиоглитазон, и 28\% больных, принимающих плацебо. У пациентов, принимающих препарат, отмечены уменьшение гликемии натощак, через 2 ч после нагрузки глюкозой, диастолического артериального давления, увеличения уровня ЛПВП в сравнении с больными, принимавшими плацебо [3]. В нашей работе на фоне терапии пиоглитазоном состояние углеводного обмена улучшилось у всех подгрупп пациентов. У пациентов с полиморфизмом rs1801282 с нарушением толерантности к глюкозе влияние терапии на показатели углеводного обмена было выражено сильнее, чем у пациентов с диким типичным генотипом PPAR - $\gamma$. Тот факт, что у пациентов с СД2 не получено данных за статистически значимое влияние полиморфизма rs1801282 на результаты применения пиоглитазона, однако отмечалась тенденция к большему снижению глюкозы плазмы натощак в случае носительства полиморфного гена, также заслуживает внимания 
и дальнейшего изучения. Таким образом, в исследовании было показано, что активность пиоглитазона зависит от наличия полиморфизма rs1801282.

Результаты нескольких исследований в схожих популяциях больных не противоречат таковым в настоящей работе: при назначении пиоглитазона или росиглитазона имел место больший эффект у пациентов-носителей rs1801282 [6-10].

В то же время имеется ряд исследований, указывающих на то, что лица с полиморфизмом имеют лишь незначительное преимущество перед остальными пациентами в отношении снижения гликемии на фоне терапии тиазолидиндионами [11, 12]. Так, Namvaran F. и соавт. включили 101 пациента с СД2. Назначение пиоглитазона в дозе 15 мг/сут не приводило к статистически значимому улучшению результатов применения препарата в подгруппе пациентов-носителей полиморфизма rs1801282. В исследовании Blüher М. и соавт. назначение пиоглитазона в дозе 45 мг/сут не вызывало увеличения вероятности ответа на пиоглитазон у носителей полиморфизма rs1801282, определяемого как уменьшение гликемии натощак более чем на 20\% или уменьшение $\mathrm{HbA}_{1 \mathrm{c}}$ более чем на $15 \%$.

Неоднородность выводов приведенных работ может быть обусловлена несколькими факторами.

Во-первых, с учетом того, что эффекты rs1801282 могут зависеть от этнической принадлежности больного $[13,14]$, следует обратить внимание на то, что приведенные исследования проводились в разных странах: в Kopee [7], Китае и Тайване [6, 8], Индии [9], Иране [12], Мексике [10] и Германии [11]. В настоящей работе принимали участие пациенты, относящиеся к однородной этнической группе, эффекты полиморфизма у которых могут отличаться от таковых в других этносах.

Во-вторых, стоит отметить, что в большинстве исследований количество пациентов с полиморфизмом было невелико. Нельзя исключить, что ряд несоответствий в результатах приведенных работ носит случайный характер. Также стоит отметить методологические различия исследований. Например, Blüher M. и соавт. использовали специфические критерии ответа на пиоглитазон, данный критерий эффективности мог иметь более низкую чувствительность в сравнении с абсолютными значениями гликемии. Другим примером могут служить низкие дозы пиоглитазона в исследовании Namvaran F. и соавт.

В-третьих, важным представляется факт включения в наше исследование больных с НТГ. В вышеупомянутые работы такие пациенты не включались. Тем не менее полученные нами данные свидетельствуют, что именно у этой категории больных имела место наиболее выраженная зависимость эффекта пиоглитазона на гликемический профиль от наличия полиморфизма rs1801282. Количество пациентов с предиабетом увеличивается, поэтому представляется актуальным поиск новых, патогенетически обоснованных фармакологических групп препаратов, положительно влияющих на состояние углеводного обмена. В настоящее время более частое назначение тиазолидиндионов у пациентов с НТГ невозможно, так как это всегда off-label исследование, что требует более трудоемкого подхода. По-прежнему для лечения таких пациентов препаратом первой линии остается метформин, хотя эффекты глитазонов также направлены на коррекцию нескольких компонентов МС.

\section{Ограничения исследования}

Настоящее исследование имеет ряд ограничений. Наиболее важным из них представляется небольшое количество пациентов с полиморфизмом rs1801282, страдающих нарушениями углеводного обмена. Другим важным ограничением является то, что в исследовании принимали участие больные с впервые выявленным MC, которым при установлении диагноза назначались не только пиоглитазон, но и проведение мероприятий по модификации образа жизни. Дополнительные факторы теоретически могут оказывать различное влияние на пациентов с полиморфизмом rs 1801282 и без него, что также могло сказаться на результатах исследования.

\section{ЗАКЛЮЧЕНИЕ}

Одним из актуальных направлений современной медицины, безусловно, следует считать персонифицированный подход в терапии различных заболеваний. Реализация «пациентоориентированной» модели невозможна без учета имеющихся полиморфизмов, влияющих на клиническое течение заболевания и эффективность применения различных препаратов. Терапия ранних нарушений углеводного обмена и СД2 является классической моделью для поиска новых фармакогенетических маркеров. Учитывая, что у одной трети пациентов терапия глитазонами является неэффективной [3] следует усилить поиск генетических детерминант, которые будут надежными прогностическими векторами эффективного лечения пациентов.

Результаты настоящей работы наглядно демонстрируют, что максимальный гипогликемический эффект от применения глитазонов наблюдался в группе пациентов с НТГ, носителей полиморфного аллеля rs1801282. Безусловно, также заслуживает внимания установленная тенденция к большему снижению гликемии у пациентов с СД2, носителей указанного полиморфного аллеля. Целесообразно проведение более крупных и длительных клинических исследований для изучения эффективности и безопасности применения пиоглитазона у пациентов с нарушениями углеводного обмена при носительстве полиморфизма rs1801282.

\section{ДОПОЛНИТЕЛЬНАЯ ИНФОРМАЦИЯ}

Источник финансирования. Подготовка и публикация рукописи проведены на личные средства авторского коллектива.

Конфликт интересов. Авторы декларируют отсутствие явных и потенциальных конфликтов интересов, связанных с публикацией настоящей статьи.

Участие авторов. Еременко Т.В. - концепция и дизайн исследования, сбор и статистическая обработка материала, анализ полученных данных, написание текста; Мациевский Н.А. - концепция и дизайн исследования, анализ полученных данных; Ворохобина Н.В. - концепция и дизайн исследования, анализ полученных данных; Матезиус И.Ю. концепция исследования, анализ полученных данных, написание текста; Абрамашвили И.Н. - анализ полученных данных, статистическая обработка материала. Все авторы внесли значимый вклад в проведение исследования и подготовку статьи, прочли и одобрили финальную версию статьи перед публикацией. 


\section{СПИСОК ЛИТЕРАТУРЫ | REFERENCES}

1. Lehrke M, Lazar MA. The many faces of PPARgamma. Cell. 2005;123(6):993-999. doi: https://doi.org/10.1016/j.cell.2005.11.026.

2. Umpierrez G, Dagogo-Jack S. Role of thiazolidinediones in the management of type 2 diabetes: focus on ethnic minority populations. Ethn Dis. 2006;16(1):51-57.

3. DeFronzo RA, Tripathy D, Schwenke DC, et al. Pioglitazone for diabetes prevention in impaired glucose tolerance. N Engl J Med. 2011;364(12):1104-1115. doi: https://doi.org/10.1056/nejmoa1010949.

4. Galbete C, Toledo E, Martínez-González MA, et al. Pro12Ala variant of the PPARG2 gene increases body mass index: An updated meta-analysis encompassing 49,092 subjects. Obesity (Silver Spring). 2013;21(7):1486-1495. doi: https://doi.org/10.1002/oby.2015.

5. Alberti KGMM, Eckel RH, Grundy SM, et al. Harmonizing the metabolic syndrome: a joint interim statement of the International Diabetes Federation Task Force on Epidemiology and Prevention; National Heart, Lung, and Blood Institute; American Heart Association; World Heart Federation; International Atherosclerosis Society; and International Association for the Study of Obesity. Circulation. 2009;120(16):1640-1645. doi: https://doi.org/10.1161/CIRCULATIONAHA.109.192644.

6. Blüher M, Lübben G, Paschke R. Analysis of the relationship between the Pro12Ala variant in the PPAR-gamma2 gene and the response rate to therapy with pioglitazone in patients with type 2 diabetes. Diabetes Care. 2003;26(3):825-831. doi: https://doi.org/10.2337/diacare.26.3.825.

7. Hsieh MC, Lin KD, Tien KJ, et al. Common polymorphisms of the peroxisome proliferator-activated receptorgamma (Pro12Ala) and peroxisome proliferatoractivated receptor-gamma coactivator-1 (Gly482Ser) and the response to pioglitazone in Chinese patients with type 2 diabetes mellitus. Metabolism. 2010;59(8):1139-1144. doi: https://doi.org/10.1016/j.metabol.2009.10.030.
8. Kang ES, Park SY, Kim HJ, et al. Effects of Pro12Ala polymorphism of peroxisome proliferator-activated receptor gamma2 gene on rosiglitazone response in type 2 diabetes. Clin Pharmacol Ther. 2005;78(2):202-208. doi: https://doi.org/10.1016/j.clpt.2005.04.013.

9. Pei $Q$, Huang $Q$, Yang $G$, et al. PPAR- $\gamma 2$ and PTPRD gene polymorphisms influence type 2 diabetes patients' response to pioglitazone in China. Acta Pharmacol Sin. 2013;34(2):255-261. doi: https://doi.org/10.1038/aps.2012.144

10. Priya SS, Sankaran R, Ramalingam S, et al Genotype Phenotype Correlation of Genetic Polymorphism of PPAR Gamma Gene and Therapeutic Response to Pioglitazone in Type 2 Diabetes Mellitus- A Pilot Study. J Clin Diagn Res. 2016;10(2):FC11-FC14. doi: https://doi.org/10.7860/jcdr/2016/16494.7331.

11. Ramírez-Salazar M, Pérez-Luque E, FajardoAraujo M, et al. Effect of the Pro12Ala polymorphism of the PPAR gamma 2 gene on response to pioglitazone treatment in menopausal women. Menopause. 2008;15(6):1151-1156. doi: https://doi.org/10.1097/gme.0b013e31816d5b2d.

12. Namvaran F, Azarpira N, Rahimi-Moghaddam P, Dabbaghmanesh MH. Polymorphism of peroxisome proliferatoractivated receptor $\gamma$ (PPARY) Pro12Ala in the Iranian population: relation with insulin resistance and response to treatment with pioglitazone in type 2 diabetes. Eur J Pharmacol. 2011;671(1-3):1-6. doi: https://doi.org/10.1016/j.ejphar.2011.09.158.

13. Mtiraoui N, Turki A, Nemr R, et al. Contribution of common variants of ENPP1, IGF2BP2, KCNJ11, MLXIPL, PPARY, SLC30A8 and TCF7L2 to the risk of type 2 diabetes in Lebanese and Tunisian Arabs. Diabetes Metab. 2012;38(5):444-449. doi: https://doi.org/10.1016/j.diabet.2012.05.002.

14. Wang $X$, Liu J, Ouyang $Y$, Fang $M$, et al. The association between the Pro12Ala variant in the PPARY2 gene and type 2 diabetes mellitus and obesity in a Chinese population. PLoS One. 2013;8(8):e71985. doi: https://doi.org/10.1371/journal.pone.0071985.

\section{ИНФОРМАЦИЯ ОБ АВТОРАХ [AUTHORS INFO]:}

*Еременко Татьяна Викторовна [Tatyana V. Eremenko, MD]; адрес: 195067, Россия, Санкт-Петербург, Пискаревский пр., д. 47 [address: 47 Piskarevskij prospect, 195067 St. Petersburg, Russia]; ORCID: https://orcid.org/0000-0002-2279-6324; eLibrary SPIN: 4499-4789; e-mail: eremenkotanja2007@rambler.ru

Мациевский Николай Александрович, к.м.н. [Nikolay A. Matsievskiy, MD, PhD];

ORCID: https://orcid.org/0000-0003-4486-4778; eLibrary SPIN: 7884-5099; e-mail: nickomedicus@mail.ru

Ворохобина Наталья Владимировна, д.м.н., професcop [Natalya V. Vorokhobina, MD, PhD, Professor];

ORCID https://orcid.org/0000-0002-9574-105X; eLibrary SPIN:4062-6409; e-mail natvorokh@mail.ru

Матезиус Ирина Юрьевна, к.м.н. [Irina Y. Matesius, MD, PhD]; ORCID: https://orcid.org/0000-0002-8694-9756;

eLibrary SPIN: 7421-1610; e-mail: Irina.Matezius@szgmu.ru

Абрамашвили Ирина Николаевна, студент [Irina N. Abramashvili, student]

ORCID: https://orcid.org/0000-0002-8142-5304; eLibrary SPIN: 7648-8589 e-mail: irina-300697@yandex.ru

*Автор, ответственный за переписку / Corresponding author.

\section{ЦИТИРОВАТЬ:}

Еременко Т.В., Мациевский Н.А., Ворохобина Н.В., Матезиус И.Ю., Абрамашвили И.Н. Клинический эффект применения тиазолидиндионов у пациентов с нарушениями углеводного обмена при носительстве полиморфизма rs1801282 // Ожирение и метаболизм. - 2020. - Т. 17. - №2. - С. 193-199. doi: https://doi.org/10.14341/omet9943

\section{TO CITE THIS ARTICLE:}

Eremenko TV, Matsievskiy NA, Vorokhobina NV, Matezius IY, Abramashvili IN. Clinical effect of thiazolidinediones in subjects with disorders of carbohydrate metabolism in case of polymorphism rs 1801282. Obesity and metabolism. 2020;17(2):193-199. doi: https://doi.org/10.14341/omet9943 